\title{
ESTUDO COMPARATIVO DOS MÉTODOS COPROLÓGICOS DE LUTZ, KATO-KATZ E FAUST MODIFICADO *
}

\author{
Adelú Chaves ** \\ Olinda Siqueira de Alcantara*** \\ Omar dos Santos Carvalho** \\ José Soares dos Santos**
}

\begin{tabular}{l|l|}
\cline { 2 - 2 } & RSPUB9/479 \\
\hline
\end{tabular}

Chaves, A. et al. Estudo comparativo dos métodos coprológicos de Lutz, Kato-Katz e Faust modificado. Rev. Saúde públ., S. Paulo, 13:348-52, 1979.

RESUMO: Foram estudados, comparativamente, em 500 pacientes, os métodos de exames de fezes de Lutz, Faust modificado e Kato-Katz para o diagnóstico parasitológico de fezes. $O$ método de Kato-Katz proporcionou maiores indices de positividade do que as outras duas técnicas no diagnóstico de ancilostomideos, T. trichiurus $e$ S. mansoni. Nenhuma diferença foi observada quanto ao diagnóstico de A. lumbricoides. Para o diagnóstico das protozooses não houve diferença significativa entre os métodos de Faust modificado e de Lutz. Baseado nos dados obtidos, eficiência, simplicidade e rapidez de execução recomenda-se a associação dos métodos de Kato-Katz e Faust modificado na rotina de diagnóstico parasitológico de fezes.

UNITERMOS: Diagnósticos de laboratório. Doenças parasitárias.

\section{N T RODU C A O}

Apesar da existência de inúmeros métudos, quantitativos e qualitativos, propostos para o exame parasitológico de fezes, todos têm sido objeto de criticas as mais variadas. quer pela complexidade e baixa sensibilidade, quer pelo elevado custo de execução, restringindo suas utilizações na rotina laboratorial de exame de fezes.

Vários autores como Brener e col. ". Chaia e col.2, Coura e Conceição ", Faust e col. $^{+}$, Ferreira 5 , Katz e Chaia ${ }^{8}$, Kato $\&$ Miura ', Maldonado e col.12, Mello e col. ${ }^{13}$, têm-se ocupado em estudar comparativa- mente a eficácia dos diferentes métodos até hoje descritos para o diagnóstico parasitológico de fezes.

Por outro lado, a Organizaçāo Mundıal da Saúde ${ }^{14}$, recomenda sobre a necessidade de utilização de métodos quantitativos em inquéritos epidemiológicos.

No presente trabalho. faz-se o estudo comparativo entre os métodos de sedimentação espontânea de Lutz"11 ("redescoberto" por Hoffman e col. ${ }^{6}$ ), quantitativo de Kato modificado por Katz e col. ${ }^{9}$, e o de concen-

* Parcialmente subvencionato pelo CNPq - PDE 02-2-07.

* Do Centro de Pesquisas "René Rachou"/FIOCRUZ - Av. Augusto de Lima. 1715 - 30000 - Belo Horizonte, MG - Brasil.

** Do INAMPS - Laboratório Central - Belo Horizonte, MG - Brasil. 
CHAVES, A. et al, Estudo comparativo dos métodos coprológicos de Lutz. Kato-Katz e Faust modificado. Rev. Saúde públ., S. Paulo, 13:348-5z: 1979.

tração pelo sulfato de zinco de Faust modificado por Larsh ${ }^{10}$.

\section{MATERIAL E METODOS}

\section{Amostra}

Foram examinadas 500 amostras fecais, oriundas na sua totalidade de segurados do Instituto Nacional de Assistência Médicá e Previdência Social (INAMPS), que procuraram o Laboratório Central deste órgão em Belo Horizonte.

\section{Exame parasitológico de fezes}

Cada amostra foi examinada concomitantemente pelos métodos de Lutz, Kato-Katz e Faust mod. Para cada método foi examinada uma lâmina do mesmo material.

Os exames utilizando-se o método de Lutz foram realizados pelos funcionários do próprio Laboratório Central, enquanto as lâminas preparadas pelos métodos de Kato-Katz e de Faust mod. foram examinadas pelos técnicos do Centro de Pesquisas "René Rachou".

Método de Faust mod. - Cerca de 2 a $4 \mathrm{~g}$ de fezes homogeneizadas são colocadas a um tubo de ensaio $(15 \times 18 \mathrm{~mm})$ contendo solução de sulfato de zinco a $33 \%$, com densidade 1.180 , até sua metade. Em seguida o tubo é colocado em um suporte e completado o seu volume com a mesma solução até formar um menisco acima de sua extremidade e, sobre o qual é colocada uma lamínula de vidro $(15 \times 24 \mathrm{~mm})$. Após 15 min. a laminula é retirada e depositada imediatamente sobre uma lâmina de vidro contendo uma gota de Iugol.

Método de Lutz - Aproximadamente 2 a $4 \mathrm{~g}$ de fezes são homogeneizadas com água e passadas para um cálice de sedimentação de $100 \mathrm{ml}$, através de "gase dobrada" em quatro, e seu volume completado, com água de torneira. Esta suspensão era mantida em repouso por 4 a 6 horas $e$ posteriormente examinada sob microscópio.

Método de Kato-Katz - Consiste em uma simplificação do método de Kato ', introduzıda por Katz e col.9* *. Sobre uma pequena amostra de fezes colocada sobre papel absorvente deposita-se uma tela de nylon que comprimida com auxílio da espátula fará com que parte das fezes passe através de suas malhas. Estas são recolhidas com a espátula e comprimidas no orifício de uma placa perfurada, que já deverá estar sobre uma lâmina, até que este se encontre cheio. Retirar o excesso de fezes com a lateral da espátula. Levantar a placa perfurada, inclinando, inicialmente, uma das extremidades e retirá-la de modo a permanecer sobre a lâmina de vidro um cilindro de amostra fecal. Sobre este cilindro é colocada uma lâmina de celofane, previamente embebida em solução de DIAFIX.

A lâmina é em seguida invertida sobre uma superfície lisa e pressionada de modo a espalhar uniformemente o material entre lâmina e lamínula evitantdo o extravasamento das fezes. Aguarda-se $30 \mathrm{~min}$. para clarificação do esfregaço fecal e examina-se ao microscópio.

A lâmina assim preparada conserva-se por cerca de um ano, sendo que para identificação de ancilostomideos e Hymenolepis nana a observação deve ser feita imediatamente após a preparação da lâmina.

\section{Análise estatistica}

Para a análise estatística dos dados, usou-se o teste do $X=$ a um nivel de significância de $5 \%$.

\section{RESULTADOS}

Observando-se os resultados dos exames de fezes pelos três métodos utilizados, veri-

* Kit AK para exame parasitológico de fezes.

AK - Indústria e comércio Ltda. - Belo Horzonte. 
CHAves, A. et al. Estudo comparativo dos métodos coprológicos de Lutz, Kato-Katz e Faust modificado. Rev. Saúde públ., S. Paulo, 13:348-52, 1979.

ficou-se que a exceção de $A$. lumbricoides, as diferenças a favor do método de Kato-Katz foram estatisticamente significativas ao nivel de $5 \%$.

Assim, para A. lumbricoides foram diagnosticados $29,2 \%$ dos casos utilizando-se o método de Lutz, enquanto $28,2 \%$ e $16,2 \%$ o foram, respectivamente, pelos métodos de Kato-Katz e Faust mod. (Tabela)

Com respeito aos ancilostomídeos, o método de Kato-Katz detectou 20,0\% dos casos, o de Faust mod. $13,8 \%$ e o de Lutz $9,0 \%$. Para $T$. trichiurus as percentagens foram de $28,8 \%, 13,6 \%$ e $7,8 \%$ utilizando- se, respectivamente, os métodos de Kato-Katz, Faust mod. e Lutz.

Com relação ao $S$. mansoni o métodu de Kato-Katz detetou $17,6 \%$ (88) dos pacientes eliminando ovos através das fezes, enquanto a utilização dos métodos de Lutz e Faust detectaram, respectivamente, apenas $7 \%$ (35) e 0,4 (2) dos indivíduos parasitados.

Por outro lado os estudos estatisticos não revelaram diferenças significativas entre os métodos de Faust mod. e Lutz para o diagnóstico das protozooses.

T A B E L A

Resultado de 500 exames parasitológicos de fezes realizados pelos métodos de Lutz, Kato-Katz e Faust mod.

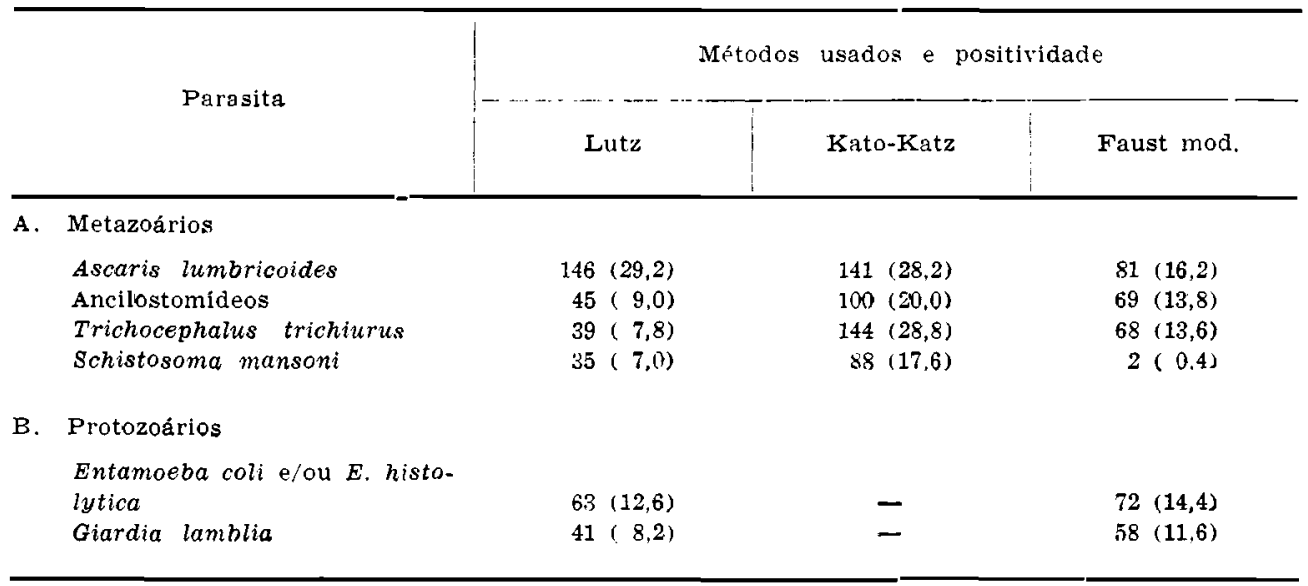

( ): percentagem

\section{DISCUSSAO E CONSLUSOES}

Os dados obtidos revelam que o método Kato-Katz apresentou uma sensibilidade (positividade) nitidamente superior aos métodos de Lutz e Faust mod. De fato aquele método revelou 2 a 4 vezes mais casos de ancilostomideos, $T$. trichiurus e $S$. mansoni, sendo, entretanto, equivalente ao método de Lutz no diagnóstico de $A$. lumbricoides.

Coura e Conceição ${ }^{3}$, comparando os métodos de Lutz, Simões Barbosa e Kato-Katz, observaram maior sensibilidade deste ültimo apesar da menor quantidade de fezes utilizada, embora com maior dispersão no seu desvio padrão. Mello e col.13, concluem existir diferença significativamente superior do método de Kato-Katz sobre o de Simōes Barbosa quanto à determinação do grau de infecção na esquistossomose, não havendo, entretanto, diferença significativa entre ambos os métodos quanto a positividade dos helmintos diagnosticados. 
CHAVES, A. et al. Estudo comparativo dos métodos coprológicos de Lutz, Kato-Katz e Faust modificado. Rev. Saude publ., S. Paulo, 13:348-52. 1979.

Em vista do grande número de coproscopias realizadas diariamente pelos laboratórios clínicos do INAMPS e de outros serviços públicos e/ou particulares, torna-se necessária a indicação de métodos de fácil execução e alta sensibilidade. Por isto mesmo, e em decorrência do baixo custo. maior sensibilidade, processamento simples e rápido, permitindo a conservação dos ovos por longo tempo e dispensando aparelhagem de difícil transporte, o método de Kato-Katz pode ser utilizado com vantagens em inquéritos epidemiológicos e na rotina laboratorial de exame de fezes para o diagnóstico de helmintos intestinais, aliado ao método de Faust mod. comprovadamente mais simples e rápido para o diagnóstico de protozooses.

Chaves, A. et al. IA comparative study of the coprologic methods of Lutz, Kato-Katz and the Faust modified.] Rev. Saúde públ., S. Paulo, 13: 348-52, 1979.

ABSTRACT: A comparative study was of the Lutz, Faust (modified), and Kato-Katz stool examination methods for parasitological diagnosis using 500 patients. The Kato-Katz method provided higher rates of positivity than the other two techniques in the diagnosis of Ancylostomidae, $\mathrm{T}$. trichiurus, and $\mathrm{S}$. mansoni. No differences were observed in the diagnosis of A. lumbicoides. Nor was a statistically significant difference observed in the diagnosis of protozoosis when using the Lutz and modified Faust methods. The data obtained led to a recommendation for an association of Kato-Katz and modified Faust methods for routine parasitological diagnosis due to its simplicity, rapidity, and efficiency.

Uniterms: Diagnosis, laboratory. Parasitic diseases.

\section{REFERENCIAS BIBLIOGRÄFICAS}

1. BRENER, $z$. et al. Valor do método de Hoffman, Pons e Janer no diagnóstico da esquistossomose mansoni. Rev. bras. Malar., 9:497-500, 1957 .

2. CHAIA, G. et al. Coprological diagnosis of Schistosomiasis. II - Comparative study of quantitative methods. Rev. Inst. Med. trop. S. Paulo, 10:349-53. 1968.

3. COURA, J. R. \& CONCEIÇAO, M. J. Estudo comparativo dos métodos de Lutz, Kato-Katz e Simões Barbosa no diagnóstico coprológico da esquistossomose mansoni. Rev. Soc, hras. Med. trop., 8:153-7. 1974

4. FAUST, E. C. et al. Comparative efficiency of various technics for the diagnosis of Protozoa and helminths in feces. J. Parasit., 25:241-62, 1939.
5. FERREIRA, I. F. O exame parasitológico das fezes. Estudo comparativo das principais técnicas. Hospital, Rio dt Janeiro, 70:347-68, 1966.

6. HOFFMAN, W. A, et al. The sedimentation-concentration method in schistosomiasis mansoni. Puerto Rico J. publ. Hlth trop. Med., 9:283-98, 1934.

7. KATO, K. \& MIURA, M. Comparative examinations. Jap. J. Parasit., $3: 3 E$ 1954.

8. KATZ. N. \& CHAIA, G. Coprological diagnosis of Schistosomiasis. I. Evaluation of quantitative technique. Rev. Inst. Med. trop. S. Paulo, 10:295-8, 1968.

9. KATZ, N. et al. A simple device for quantitative stool thick-smear technique in schistosomiasis mansoni. Rev. Inst. Med. trop. S. Paulo, 14:397-400, 1972. 
CHAVES, A. et al. Estudo comparativo dos métodos coprológicos de I utz. Kato-Katz e Faust modificado. Rev. Saúde públ., S. Paulo, 13:348-52. 1979.

10. LARSH apud PESSOA, S. B. Parasitologia médica, $10^{\text {at }}$ ed. Rio de Janeiro, Ed. Guanabara-Koogan, 1976. p. 914.

11. LUTz. A. O. Schistosomum mansoni e a Shistomatose segundo observações fejtas no Brasil. Mem. Inst. Oswaldo Cruz, 11:121-44, 1919 .

12. MALDONADO, J. F. et al. Comparative value of fecal examination procedure in the diagnosis of helminth infections. Exp. Parasit. 2:294-310, 195 ?.
13. Mello, D. A. Comparação dos métodos coprológicos quantitativos de Barbosa (1969) e Kato-Katz et al. (1972). Rev. Joiana Med., 23:53-9, 1977.

14. WORLD HEALTH ORGANIZATION. Scientific Group on Research in Bllharziasis (Chemotherapy), Geneva, 1964. Chemotherapy of Bilharzias; report. Geneva. 1966. (Techn. Rep. Ser., 317).

Recebido para publicagão em 18/06/1979 Aprovado para publicação em 30/07/1979 